\title{
Design and Simulation of a Tunable Stripline Wideband Coupler
}

\author{
Bing Luo ${ }^{1}$, Yongliang Zhang ${ }^{1}$ \\ ${ }^{1}$ School of Electronics \& Communication Engineering, Guangdong Mechanical \& Electrical College, Guangzhou 510515, China
}

\begin{abstract}
The basic theory of coupler is introduced, and a tunable strip-line wideband coupler is designed by using radio-frequency/microwave software which is named ADS on the basis of theoretical calculations and analysis. The broadband coupler can be adjusted appropriately in different frequency regions by using a tunable band line. It has a wide bandwidth of $2 \mathrm{G} \sim 4 \mathrm{G}$, excellent return loss of ( $>30.5) \mathrm{dB}$, stable coupling coefficient of $(9.85 \sim 10.81) \mathrm{dB}$, and high isolation of $(>20.08) \mathrm{dB}$, the straight-through pass-band transmission coefficient of $(0.41 \sim 0.62) \mathrm{dB}$. The coupler has high power tolerance and small size. It can reduce design costs by used in communication systems.
\end{abstract}

Keywords: Tunable; Stripline; Wideband coupler; Return loss; Coupling coefficient; Simulation and analysis.

\section{Introduction}

Coupler has always been a very important part of wireless communication system. It is often used for sampling microwave signals with specified flow direction, such as power monitoring and power gain control in microwave signal generator ${ }^{[1-4]}$, anti-interference measurement or spurious measurement in receiver. Therefore, coupler has been a hot research topic in the field of wireless communication system ${ }^{[1-5]}$. Generally speaking, the coupler design is based on the parameters of the theoretical calculation, and get the actual coupler design parameters, but this method is very tedious and not accurate. The theoretical calculation is more complex and poor performance. Few can adjust the parameters of the wideband coupler. In fact, RF/microwave simulation software is a necessary tool for coupler design to evaluate the performance of the coupler. A large-scale EDA software developed which is named Advanced Design System (ADS) by Agilent, and is one of the most popular software in microwave circuit and communication system simulation. Aiming at the complicated way of traditional theoretical calculation and design, this paper uses ADS software to design a kind of adjustable stripline wideband coupler quickly and accurately, which saves time and design cost, and reduces the cost of communication system updating and upgrading. Aiming at the problem of poor directivity of general couplers, a tunable design method is proposed. By using this method, the directivity of couplers and other parameters are greatly improved. Using this coupler in communication system can improve the detection accuracy of communication system, and improve the communication quality and effect.

\section{The design principle of wideband coupler}

As is known to all, a microstrip line is a signal line with a layer of dielectric separating from the ground plane. The other side is placed in the air. If the thickness and width of the line and the distance between the line and the ground plane are controllable, its characteristic impedance can also be controlled. A stripline is a signal line between two layers of conductive plane. If the thickness and width of the line, the dielectric constant of the medium and the distance between the two layers of conductive plane are controllable, the characteristic impedance of the line is also controllable. Obviously, the impedance of the stripline is more controllable, the transmission of the stripline is TEM wave, while the transmission of the microstrip line is quasi-TEM wave, and the microstrip line is not conducive to achieving better direction and higher bandwidth, the structure of the microstrip line and the stripline are shown in Fig.1 and Fig.2, respectively.

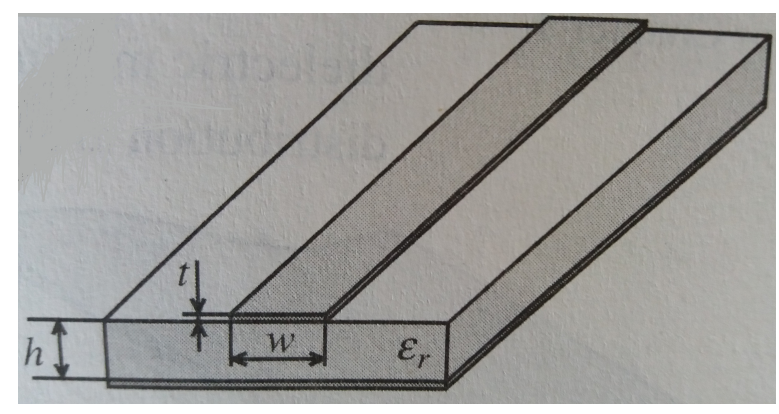

Figure 1. Microstrip structure. 


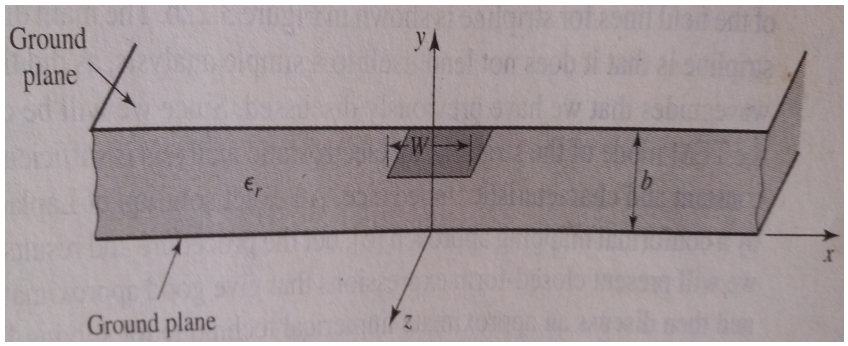

For microstrip line, the characteristic impedance can be expressed as

$$
z_{0}=\frac{60}{\sqrt{\varepsilon_{\mathrm{e}}}} \ln \left(\frac{8 d}{W}+\frac{W}{4 d}\right) \quad\left(\frac{W}{d}\right) \leq 1
$$

and

Figure 2. Stripline structure.

$$
z_{0}=\frac{120 \pi}{\sqrt{\varepsilon_{e}[W / d+1.393+0.667 \ln (W / d+1.444)]}} \quad\left(\frac{W}{d}\right)>1
$$

Where, $d$ denotes the distance between the microstrip line and the bottom horizon, $W$ denotes the width of the microstrip line. $\varepsilon_{e}$ is the effective dielectric constant of the microstrip line, and is expressed as:

$$
\varepsilon_{\mathrm{e}}=\frac{\varepsilon_{\mathrm{r}}+1}{2}+\frac{\varepsilon_{\mathrm{r}}-1}{2}
$$

$\mathcal{E}_{\mathrm{r}}$ is the dielectric constant of the dielectric layer in (3).

For stripline, the characteristic impedance can be expressed as

$$
z_{0}=\frac{30 \pi}{\sqrt{\varepsilon_{\mathrm{r}}}} \frac{b}{W_{\mathrm{e}}+0.441 b}
$$

In (4), $b$ is the thickness of the dielectric layer between the upper and lower horizons, $W_{\mathrm{e}}$ is the effective width of the stripline, $W$ is the thickness of the dielectric layer between the upper and lower horizons, and the relationship between the actual width $W$ of the stripline and the thickness $b$ of the dielectric layer between the upper and lower horizons is expressed as:

$$
\begin{aligned}
& W_{\mathrm{e}}=W \quad \frac{W}{b}>0.35 \\
& S_{11}=\frac{1}{2}\left(\frac{j \sin \theta\left(\frac{Z_{0 \mathrm{e}}}{Z_{0}}-\frac{Z_{0}}{Z_{0 \mathrm{e}}}\right)}{2 \cos \theta+j \sin \theta\left(\frac{Z_{0 \mathrm{e}}}{Z_{0}}+\frac{Z_{0}}{Z_{0 \mathrm{e}}}\right)}+\frac{j \sin \theta\left(\frac{Z_{0 \mathrm{o}}}{Z_{0}}-\frac{Z_{0}}{Z_{00}}\right)}{2 \cos \theta+j \sin \theta\left(\frac{Z_{0 \mathrm{o}}}{Z_{0}}+\frac{Z_{0}}{Z_{00}}\right)}\right. \\
& S_{21}=\frac{1}{2 \cos \theta+\mathrm{j} \sin \theta\left(\frac{Z_{0 \mathrm{e}}}{Z_{0}}+\frac{Z_{0}}{Z_{0 \mathrm{e}}}\right)}+\frac{1}{2 \cos \theta+\mathrm{j} \sin \theta\left(\frac{Z_{0 \mathrm{o}}}{Z_{0}}+\frac{Z_{0}}{Z_{0 \mathrm{o}}}\right)} \\
& S_{31}=\frac{1}{2}\left(\frac{j \sin \theta\left(\frac{Z_{0 \mathrm{e}}}{Z_{0}}-\frac{Z_{0}}{Z_{0 \mathrm{e}}}\right)}{2 \cos \theta+\mathrm{j} \sin \theta\left(\frac{Z_{0 \mathrm{e}}}{Z_{0}}+\frac{Z_{0}}{Z_{0 \mathrm{e}}}\right)}-\frac{j \sin \theta\left(\frac{Z_{0 \mathrm{o}}}{Z_{0}}-\frac{Z_{0}}{Z_{0 \mathrm{o}}}\right)}{2 \cos \theta+\mathrm{j} \sin \theta\left(\frac{Z_{0 \mathrm{o}}}{Z_{0}}+\frac{Z_{0}}{Z_{0 \mathrm{o}}}\right)}\right. \\
& S_{41}=\frac{1}{2 \cos \theta+\mathrm{j} \sin \theta\left(\frac{Z_{0 \mathrm{e}}}{Z_{0}}+\frac{Z_{0}}{Z_{0 \mathrm{e}}}\right)}-\frac{1}{2 \cos \theta+\mathrm{j} \sin \theta\left(\frac{Z_{0 \mathrm{o}}}{Z_{0}}+\frac{Z_{0}}{Z_{0 \mathrm{o}}}\right)}
\end{aligned}
$$




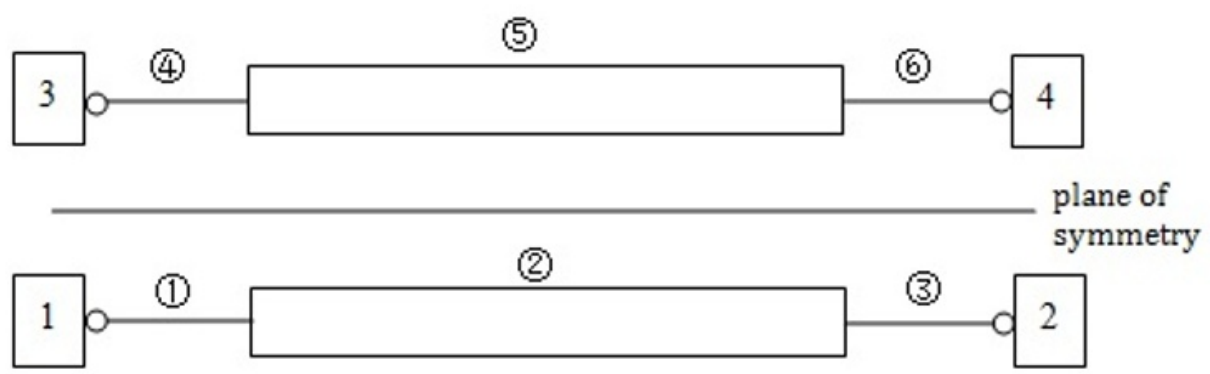

Figure 3. Single coupler structure.

The relationship between the characteristic impedance and its odd mode impedance and even mode impedance is:

$$
Z_{0}=\sqrt{Z_{0 \mathrm{o}} Z_{0 \mathrm{e}}}
$$

Therefore, by using (1), (2), and (11) can accurately calculate the structure size of the coupler.

\section{Design and simulation of a tunable stripline wideband coupler}

Based on the design principle of wideband coupler, in order to achieve wider bandwidth, a wideband coupler is designed and simulated in the form of 4-order asymmetric structure as shown in Fig.4. The parameters of wideband coupler are as follows.

(1) Bandwidth frequency is $350-2700 \mathrm{MHz}$;

(2) Insertion loss within band: less than $0.5 \mathrm{~dB}$;

(3) Coupling loss: $(9.8 \sim 10.8) \mathrm{dB}$;

(4) Isolation loss: $>20 \mathrm{~dB}$;

(5) Return loss: $>25 \mathrm{~dB}$;

(6) Input and output impedance: $50 \mathrm{ohm}$.

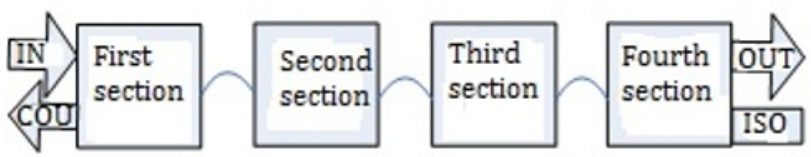

Figure 4. Structure of wideband asymmetric coupler.

The structure equivalent lumped parameters of the wideband coupler are simulated in ADS software, and then the electromagnetic simulation model of the ADS software is established. As shown in Fig. 5, the return loss (S11), insertion loss (S21), coupling loss (S31) and isolation degree (S41) of the final simulation are shown in Fig.6, Fig.7, Fig.8 and Fig.9.

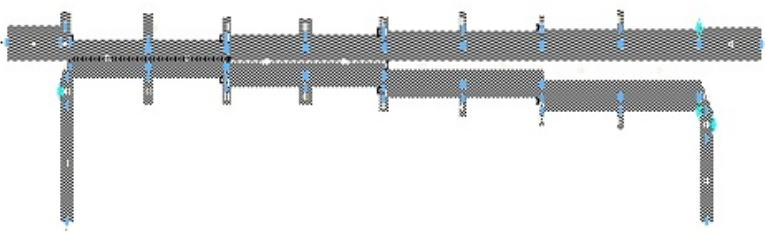

Figure 5. Electromagnetic simulation model of 4 order asymmetrical wideband coupler.

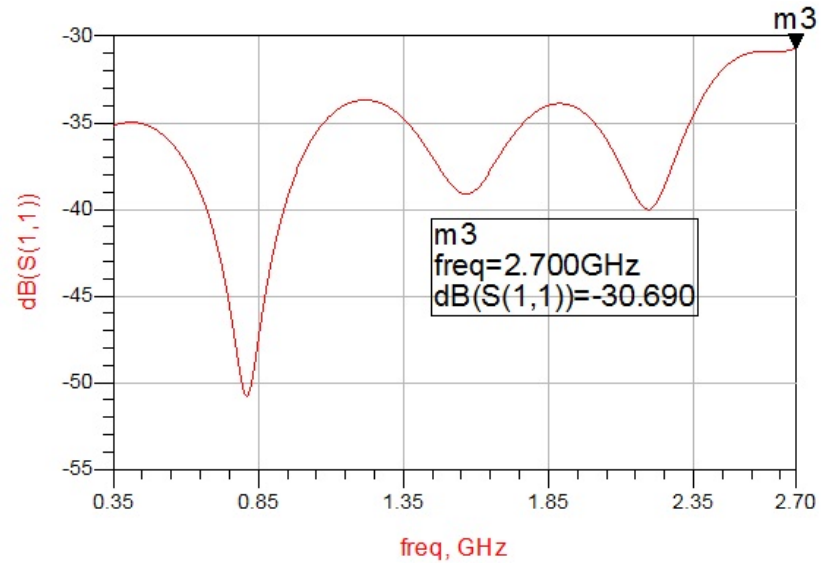

Figure 6. Simulation return loss of wideband coupler (S11).

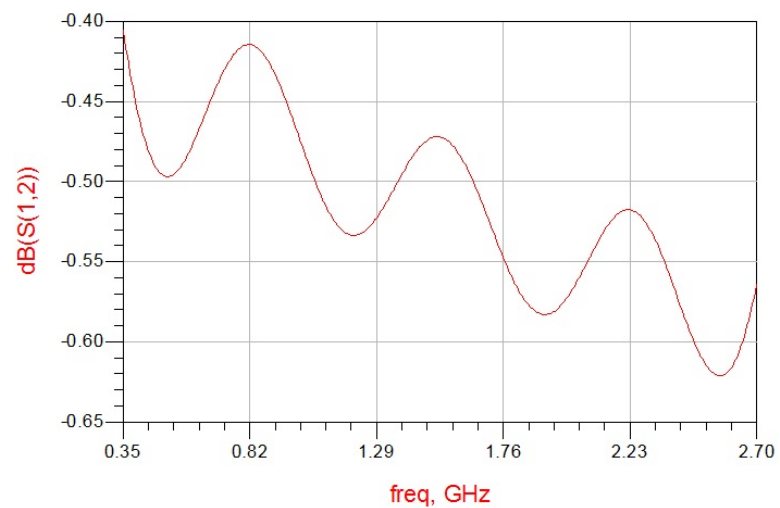

Figure 7. Simulation insertion loss of wideband coupler(S12).

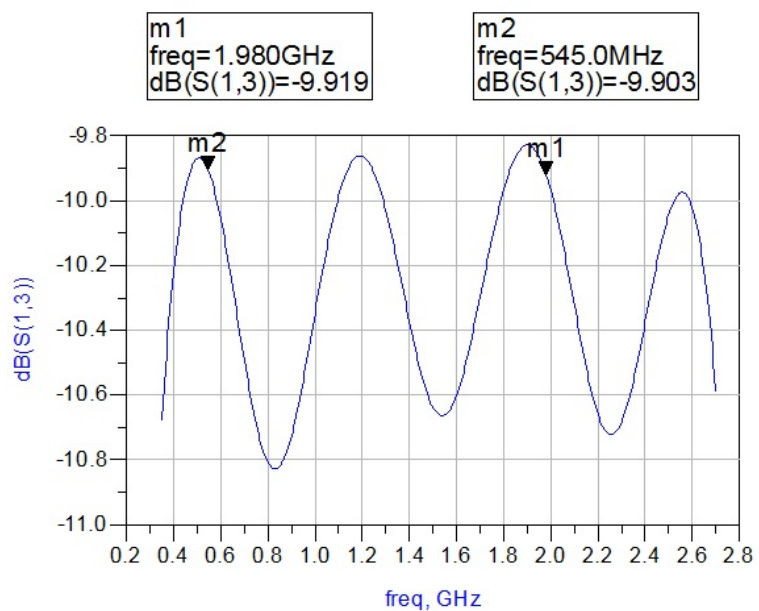

Figure 8. Simulation coupler coupling loss of wideband (S13). 


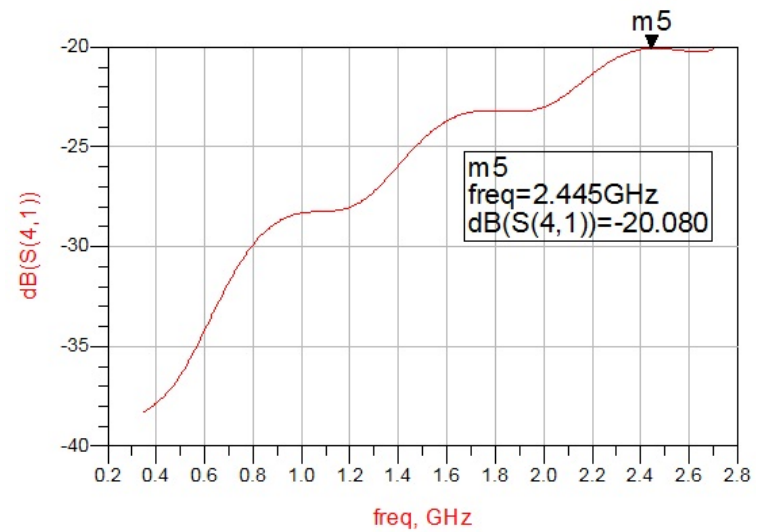

Figure 9. Simulation isolation loss of wideband coupler (S41).

\section{Comparison of simulation and practical parameters of wideband coupler}

In order to ensure the qualified rate of the product, a tunable wideband coupler is designed. The final profile of the wideband coupler is shown in Fig.10, the front and the bottom perspective are as shown in Fig.11.

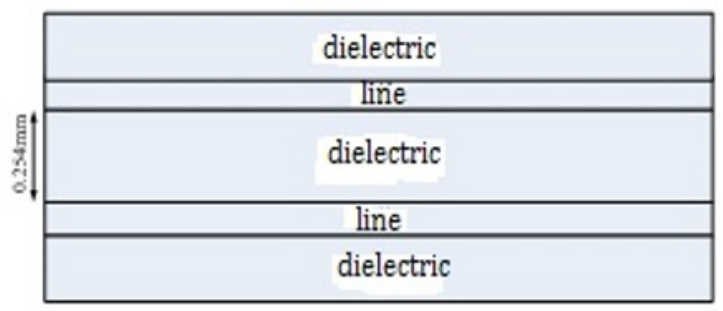

Figure 10. Profile of wideband coupler.

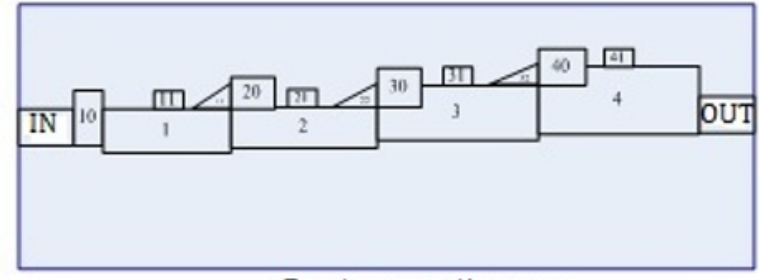

Front perspective map

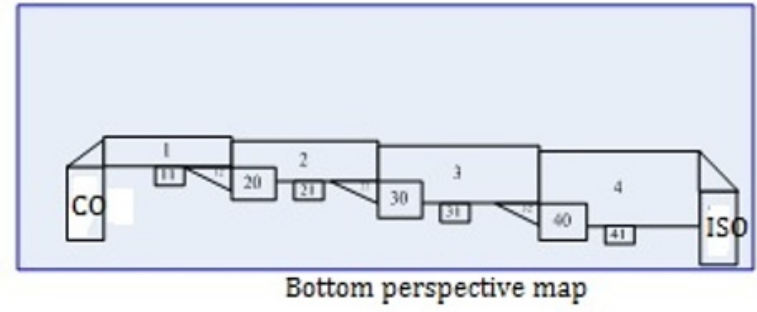

Figure 11. Front and bottom perspective map.

The parameters of the actual wideband coupler are tested by the network analyzer and compared with the simulation results. Under the condition of no tune, the return loss between the actual test and the simulation results is about $2 \mathrm{~dB}$, while the coupling loss between the actual test and the simulation results is about $0.5 \mathrm{~dB}$. By minutely adjusted the size of the stripline, the final physical test is consistent with the simulation results. Therefore, the wideband coupler can be manufactured according to the simulation results, and the design cost can be reduced.

\section{Conclusions}

Coupler design is a popular research technology in the field of communication. The traditional theoretical design method has become time-consuming and laborious. The theoretical calculation method has a large workload and low precision. Therefore, this paper uses the classical simulation software ADS to design and simulate the wideband coupler. Real design work can quickly realized. In this paper, a tunable stripline wideband coupler is used to realize, which has a wide bandwidth (covering $2 \mathrm{G}, 3 \mathrm{G}$, $4 \mathrm{G}$ and other frequency bands), excellent return loss (S11 $<-30.5 \mathrm{~dB})$, stable coupling $(-9.85 \mathrm{~dB}<\mathrm{S} 13<-10.81 \mathrm{~dB})$, high isolation $(\mathrm{S} 41<-20.08 \mathrm{~dB})$, and small in-band fluctuation $(-0.41 \mathrm{~dB}<\mathrm{S} 12<-0.62 \mathrm{~dB})$ at the straightthrough pass-band transmission port. It can be tuned in a certain range. It has high power tolerance and small size. It can be used in communication system to reduce the design cost.

\section{Acknowledgments}

The authors would like to thank 2017 Guangdong Mechanical \& Electrical College Education and Teaching Reform Fund (Research on development and practice teaching of high speed circuit board design course), 2016 Guangdong Mechanical \& Electrical College Natural Science Fund (Development of intelligent garbage bin in autonomous navigation room) and Guangdong Province Educational Science Planning Subject - Research and practice of artisan spirit leading the training mode of applied electronic technology professionals in the new era (2017GGXJK006) for their support in this research.

\section{References}

1. Liu Liang, GUO Jian. Design and Analysis of a Wideband 3 dB Coupler, Radio Engineering, vo1.43, no.6 (2013).

2. MO Zuo -jian, YA NG Kai, BU Shi -rong, ZH ENG Cheng. The Design of $\mathrm{S}$-band Broadband Directional Coupler. GUIDANCE \& FUZE, vol.30 no.4, Dec., 2009.

3. Lan Yonghai. Design of an Ultra-wideband (UWB) Multi-section Directional Coupler. Telecommunication Engineering, vol.49, no.1, Jan.2009.

4. Wang Sheng. Simulation and design of a chip LTCC coupler. J Magn Mater Devices, vol.43, no.5, (2012).

5. David M.Pozar. Microwave Engineering [M]. Publishing House of Electronics Industry, 2006, 9: 139, 144-145. 